Article

\title{
Design and Experimental Verification of a PZT Pump with Streamlined Flow Tubes
}

\author{
Ming Tang ${ }^{1}$, Qibo Bao ${ }^{1}$, Jianhui Zhang ${ }^{1, *}$, Qingshuang Ning ${ }^{1}$, Chaobin Chen ${ }^{1}$, Jun Huang ${ }^{2} \mathbb{C}$ \\ and Chuanyu $\mathrm{Wu}^{3}$ \\ 1 College of Mechanical and Electrical Engineering, Guangzhou University, Guangzhou 510006, China; \\ gzdxtangming@outlook.com (M.T.); baoqibo1994@outlook.com (Q.B.); ningqs@163.com (Q.N.); \\ chen_chaobin@outlook.com (C.C.) \\ 2 National Research Center of Pumps, Jiangsu University, Zhenjiang 212013, China; huangjun@ujs.edu.cn \\ 3 Faculty of Mechanical Engineering \& Automation, Zhejiang Sci-Tech University, Hangzhou 310018, China; \\ cywu@zstu.edu.cn \\ * Correspondence: zhangjh@nuaa.edu.cn
}

Received: 6 August 2019; Accepted: 10 September 2019; Published: 16 September 2019

Abstract: In this paper, a streamlined flow tube valveless piezoelectric pump (SLFT PZT pump) is proposed to modify the single flow trend and improve the fluid flow stability. Firstly, the structural and working principle of the streamlined flow tube, which accounts for changing the flow trend and improving the flow stability, were analyzed. The flow resistance and flow rate equations were established. Secondly, the pressure and velocity fields of the tube were simulated. These simulated results were consistent with the theoretical results. Thirdly, the flow resistance of the flow tube was tested with pressure differences of $1000 \mathrm{~Pa}, 1200 \mathrm{~Pa}, 1400 \mathrm{~Pa}$ and $1600 \mathrm{~Pa}$ respectively. The trend of the result curves was consistent with the simulated results. The amplitude-frequency relationship and the flow-rate-frequency relationship were also tested, both result curves highly corelate. The maximum amplitude was $0.228 \mathrm{~mm}(10 \mathrm{~Hz}, 120 \mathrm{~V})$, and the maximum flow rate was $17.01 \mathrm{~mL} / \mathrm{min}$ $(10 \mathrm{~Hz}, 100 \mathrm{~V})$. Finally, the theoretical flow rate of the SLFT PZT pump was calculated at $100 \mathrm{~V}$ and $120 \mathrm{~V}$. These results roughly fitted with the experimental results. The streamlined flow tube could change the internal flow trend that remarkably improved the flow stability. Therefore, it promoted the application of the valveless PZT pump in living cells, biomedical and polymer delivery.

Keywords: valveless piezoelectric pump; streamline structure; flow trend; flow stability

\section{Introduction}

As a functional material, piezoelectric ceramics play an irreplaceable role in modern intelligent industry [1-3]. Piezoelectric ceramics have been widely used in modern intelligent devices, such as pressure sensors [4], vibration energy collecting devices [5], intelligent wearable devices with human-computer interaction [6], and so on. At the same time, with the advent of MEMS (Micro-Electro-Mechanical System), the inverse piezoelectric driving technology that converts electrical energy into mechanical energy has become a research hotspot in research concerning modern micromachines $[7,8]$. The actuator has not only decreased in size but also enhances conversion efficiency in ultrasonic motors [9], ultrasonic transducers [10], piezoelectric pumps [11], and so on. Among them, the piezoelectric pump is further divided into valve piezoelectric pumps and valveless piezoelectric pumps.

As an emerging piezoelectric-driving-fluid technology, the valveless piezoelectric (PZT) pump has shown ever-increasing application potential in aerospace, biomedical, microfluidics and sensor fields [12-14]. It has many advantages when compared with traditional motor pumps, like its simple structure, compact size, lack of electromagnetic interference and easy integration, 
The absence of the movable valve can decrease the probability of crushing damage of the transported substance. Thus, the valveless PZT pump has been applied to clinical medical drug delivery and biological laboratories [15-17]. At the same time, the delivery characteristics of valveless PZT pumps are mainly determined by their flow tube structure. Different flow tube structures would change the flow field tremendously, and therefore would be used in different applications. The innovation of flow tube structure has become a new direction of valveless piezoelectric pump research $[18,19]$.

In 1993, E. Stemme et al. historically proposed the first cone-shaped flow tubes valveless PZT pump for fluid delivery. Since then many scholars in the field have begun to delve into further research on valveless PZT pumps with cone-shaped flow tubes [20,21]. In 2011, P. Verma. et al. designed a valveless PZT pump with 3D cone-shaped flow tubes to study the influence of excitation voltage and geometric parameters on the performance of piezoelectric ceramic [22]. In 2015, X. He et al. systematically studied cone-shaped flow tubes with different angles, to optimize the valveless PZT pump [23,24]. In addition to the traditional cone-shaped flow tube valveless PZT pump, other types of valveless PZT pump have also been widely studied. In 1995, F.K. Forster et al. used unique structure called the "Tesla valve", which has been proved to be more suitable for micro particles delivery, as the fluid channel of a PZT pump [25,26]. In 2007, J.H. Zhang et al. found that the Y-shaped flow tube exhibited obvious flow resistance difference during the pump chamber compression and expansion strokes. Later, they proposed the Y-shaped flow tube valveless PZT pump for functional polymer delivery which expand the applications of the valveless PZT pump in the medical and health care [27,28]. In 2018, F.R. Munas et al. developed a valveless PZT micropump with a crossed cone-shaped flow tube, to greatly improve micro-medical equipment for human health [29]. In 2019, L. He et al. designed a valveless PZT pump with bluff-body in the pump chamber. The pump can reduce backflow and increase output flow rate, which has potential applications in the drug delivery field [30].

The application of valveless PZT pumps, in the field of fluid delivery, has been studied by many scholars over more than twenty years. However, the singularity of the flow trend, which would cause a stronger internal vortex and less flow stability, has not been preferably modified. In 2009, X.Y. Huang et al. designed a valveless PZT micropump with an acoustically featured channel structure in the pump chamber. It was reported that the channel structure can alleviate the flow instability induced by the single flow trend [31,32]. Although the pump proposed by Huang et al. can alleviate the flow instability because of the single flow trend, the structure of the pump is still the traditional conical flow tube, which cannot avoid the cone-shaped tube's disadvantages of reflux and vortex. Moreover, the pump by Huang et al. is a plane structure (2D), which would induce some unavoidable disadvantages during fluid delivery, such as bad symmetry, large system disturbance, low efficiency, and necessary consideration of the one-way flow resistance [21].

According to the above analysis, a streamlined flow tube valveless PZT pump (SLFT PZT pump) is proposed in this paper to improve the trend of fluid flow and the flow stability. The pump has a 3D structure, which can realize the special fluid delivery and energy transfer and match the energy consumption between diffuser and nozzle. The flow resistance at each point of the streamlined flow tube produces the same external force, which will minimize the internal consumption of the pump itself. In this study, firstly, the reasons for the change of flow trend caused by streamline structure were analyzed. Then, the expressions of flow resistance and flow rate were established by theoretical analysis. Finally, the performance of the pump was further studied by means of experiment and simulation, and the results obtained are discussed in this paper. 


\section{Structure and Working Principle}

\subsection{Structural Principle}

To simplify the principle, three sections were defined for the streamlined flow tube, as shown in Figure 1.

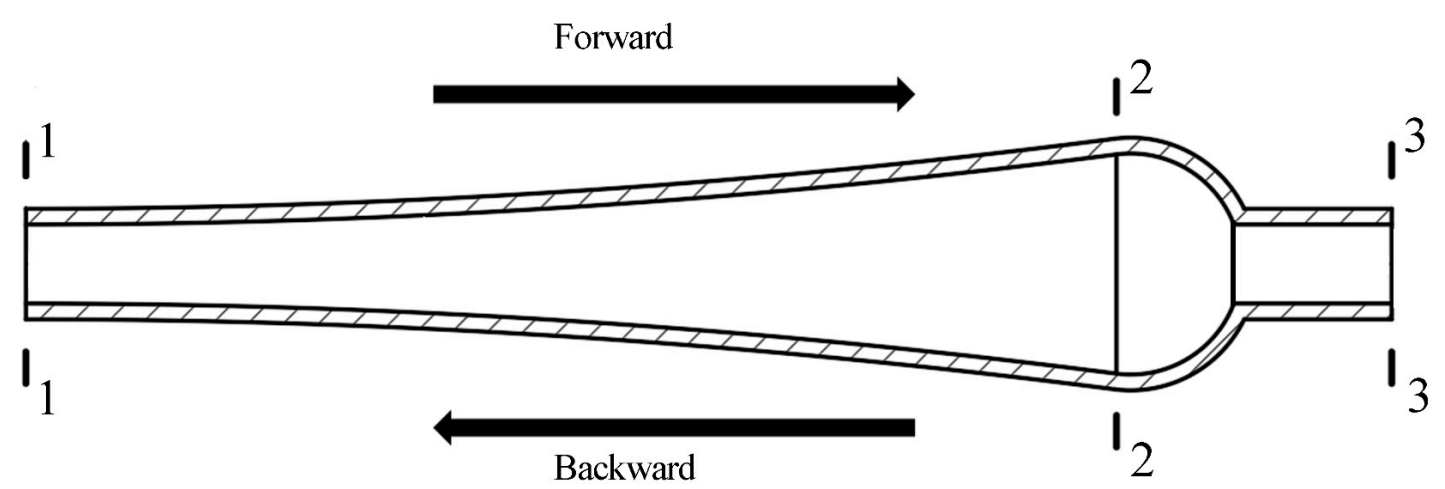

Figure 1. The section view of the streamlined flow tube.

When the fluid flows in the streamlined flow tube, the main reason for the backflow vortex is the singular flow trend of boundary layer flows, which leads to the separation of boundary layer flows [33]. Therefore, the flow trend should be fully considered when designing the flow tube. The flow tube designed consists of two parts: divergence and contraction parts. Here, forward flow direction is defined in Sections 1.1-3.3, and backward flow direction is in Sections 3.3-1.1, as shown in Figure 1. Due to fluid flows forward through the tube (positive $x$ axis), the pressure gradient changes in the boundary layer in the $x$ direction are much larger than that in normal direction ( $y$ axis). Therefore, the pressure gradient in normal direction is neglected, and the Navier-Stokes equation can be simplified as $[34,35]$

$$
\left\{\begin{array}{c}
u_{x} \frac{\partial u_{x}}{\partial x}+u_{y} \frac{\partial u_{x}}{\partial y}=-\frac{1}{\rho} \frac{\partial p}{\partial x}+v \frac{\partial^{2} u_{x}}{\partial y^{2}} \\
\frac{\partial p}{\partial y}=0 \\
\frac{\partial u_{x}}{\partial x}+\frac{\partial u_{y}}{\partial y}=0
\end{array}\right.
$$

Since the flow area from Sections 1.1-2.2 gradually increases, and the pressure gradient constantly increases, then the boundary layer equation with different pressure gradients is

$$
u_{x} \frac{\partial u_{x}}{\partial x}+\frac{\partial u_{x}}{\partial x}=-\frac{1}{\rho} \frac{\partial p}{\partial x}+v \frac{\partial^{2} u_{y}}{\partial y^{2}}
$$

When the cross-sectional area of the fluid gradually increases, it is bound to cause the pressure to increase while the flow rate decreases. According to Equations (1) and (2), it can be seen that the boundary layer fluid should satisfy the following conditions when flow area gradually increases

$$
\frac{\partial p}{\partial x}>0 \frac{\partial^{2} u_{y}}{\partial y^{2}}>0 \frac{\partial u_{x}}{\partial x}<0
$$

According to Formula (3), for a single divergent flow, the flow velocity would gradually decrease, then the boundary layer thickness would increase. When the velocity is reduced to zero, there are backflow vortexes to disturb fluid delivery.

On the contrary, when flow area gradually decreases, the boundary layer fluid should satisfy the follow expression as

$$
\frac{\partial p}{\partial x}<0 \frac{\partial^{2} u_{y}}{\partial y^{2}}<0 \frac{\partial u_{x}}{\partial x}>0
$$


According to Formula (4), for a single convergent flow the internal pressure decreases, then the flow velocity increases and the boundary thickness decreases. Therefore, the flow tube structure designed should be able to change the fluid flow trend, whose principle should obey both Equations (3) and (4), for the improvement of flow stability.

\subsection{Working Principle}

During fluid flowing in the forward direction, the initial pressure of the flow tube is $P$, the initial velocity is $u$, the radius of the flow tube at Section 2.2 is $R$, then the velocity and pressure are expressed as

$$
\left\{\begin{array}{c}
P=P(x) \\
u=u(x, y)
\end{array}\right.
$$

where $x$ is the length of the flow tube, and $y$ is the flow area radius.

Therefore, according to the formulas (1)-(4), the relationship between the pressure change and the velocity are

$$
\left\{\begin{array}{c}
\frac{d p}{d x}>0 \frac{\partial^{2} u_{y}}{\partial y^{2}}>0 \frac{\partial u_{x}}{\partial x}<0\left(0<x<L_{1-2}\right) \\
\frac{d p}{d x}<0 \frac{\partial^{2} u_{y}}{\partial y^{2}}<0 \frac{\partial u_{x}}{\partial x}>0\left(L_{1-2}<x<L_{2-3}\right)
\end{array}\right.
$$

where $L_{1-2}$ is the length from Sections 1.1-2.2, $L_{2-3}$ is the length from Sections 2.2-3.3.

During backwards fluid flow, similarly, the coordinate system is established with the Section 3.3 as the origin, the relationship between the pressure change and the velocity are

$$
\left\{\begin{array}{c}
\frac{d p}{d x}>0 \frac{\partial^{2} u_{y}}{\partial y^{2}}>0 \frac{\partial u_{x}}{\partial x}<0\left(0<x<L_{2-3}\right) \\
\frac{d p}{d x}<0 \frac{\partial^{2} u_{y}}{\partial y^{2}}<0 \frac{\partial u_{x}}{\partial x}>0\left(L_{2-3}<x<L_{1-2}\right)
\end{array}\right.
$$

According to Formulas (6) and (7), whether fluid flows forward or backward, the divergent or convergent trend is similar. For example, when fluid flows forward, the fluid begins with an adverse pressure gradient from Sections 1.1-2.2, where the internal pressure of the flow field increases while the velocity decreases. Then goes through a consequent pressure gradient from Sections 2.2-3.3, where the pressure decreases while velocity increases. Similarly, when it flows backwards, the adverse pressure gradient appears in Sections 3.3-2.2 and the consequent pressure gradient appears in Sections 2.2-1.1. The internal velocity and pressure change are opposite to the barotropic gradient. By changing the fluid flow trend, it is theoretically guaranteed that the velocity of the internal boundary layer will not appear to be zero, avoiding the occurrence of backflow and vortex, thereby improving the flow stability.

When the piezoelectric vibrator is oscillating periodically, the volume of the pump chamber changes correspondingly. Scholars have divided this periodic change into two stages: one is the suction phase (suction stroke) when the internal volume of the pump chamber increases from minimum to maximum. The other one is the discharge phase (compression stroke) when the internal volume reduces from maximum to minimum. In both the suction and compression strokes, the flow trend changes in the streamlined flow tube of the SLFT PZT pump are always opposite to each other. Thus, at any stage, there are always obvious characteristics of unequal flow resistance, which can realize macroscopic fluid transportation.

The three-dimensional model of the SLFT PZT pump, shown in Figure 2, was fabricated by Sterolithography Apparatus (iSLA660, ZRapid, Suzhou, China). The size parameters of the pump are shown in Table 1. The resonance impedance and resonance frequency of the piezoelectric vibrator (Dong Guan Cosson Electric Co, LTD, Dongguan, China) adopted in this paper are $1.9 \pm 0.5 \mathrm{KHz}$ and $400 \Omega$ respectively. The size parameters of the piezoelectric vibrator are shown in Table 2. 


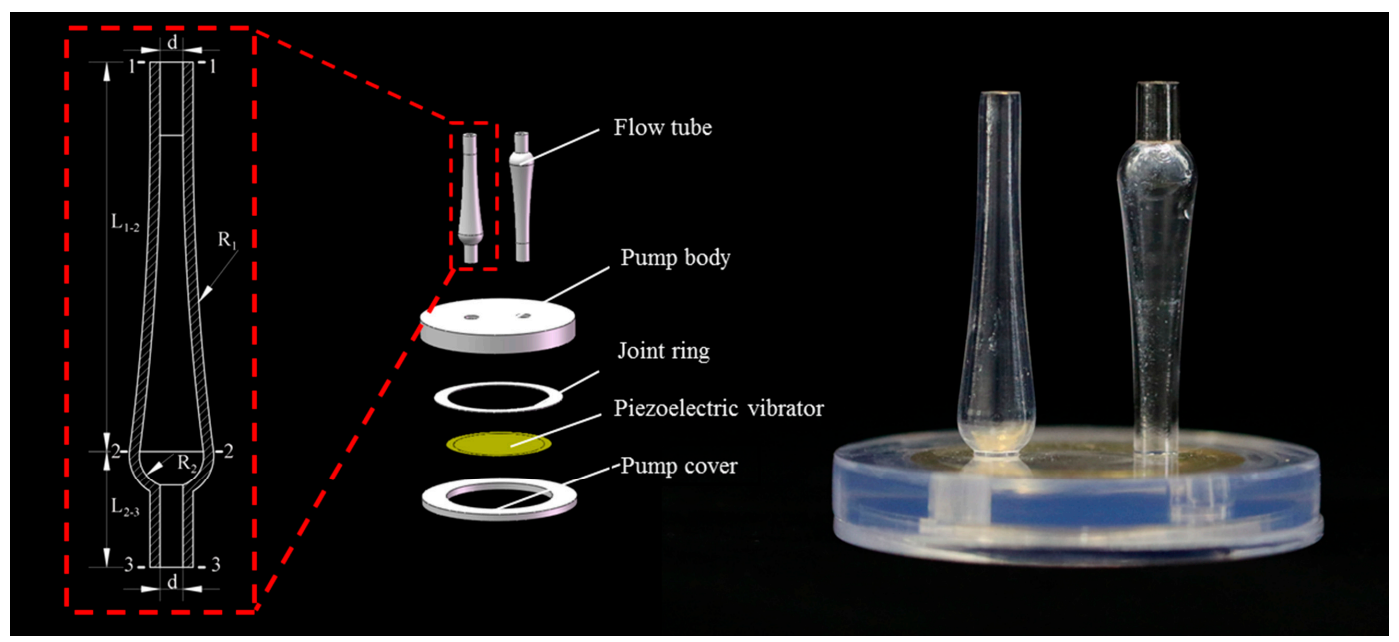

Figure 2. The streamlined flow tube valveless piezoelectric (SLFT PZT) pump.

Table 1. The size of the SLFT PZT pump.

\begin{tabular}{cc}
\hline Item & Size (mm) \\
\hline $\mathrm{d}$ & 2.5 \\
$\mathrm{~L}_{1-2}$ & 40 \\
$\mathrm{~L}_{2-3}$ & 13.8 \\
$\mathrm{R}_{1}$ & 270 \\
$\mathrm{R}_{2}$ & 4 \\
Pump chamber diameter & 35 \\
Pump chamber height & 1 \\
\hline
\end{tabular}

Table 2. The size parameters of the PZT vibrator.

\begin{tabular}{cc}
\hline Item & Size $(\mathbf{m m})$ \\
\hline Brass substrate diameter & 41 \\
Brass substrate thickness & 0.2 \\
Piezoelectric ceramics diameter & 35 \\
Piezoelectric ceramics thickness & 0.15 \\
\hline
\end{tabular}

\section{Theoretical Analysis}

In order to determine the internal pressure loss, under the conditions of mass conservation, the change of internal mass flow is independent on the flow tube size. Integrating the streamwise equation over a unit span control volume extending from 0 to $x$ yields

$$
\left(\bar{p}_{0}-\bar{p}_{x}\right) d=\rho x \frac{\partial Q}{\partial t}+\int_{0}^{x} \tau_{w}(x) d x+\rho \int_{-\frac{d}{2}}^{\frac{d}{2}}\left(u_{x}^{2}-u_{0}^{2}\right) d y
$$

where $\rho$ is the density, $\bar{p}$ is the section averaged pressure, $\tau_{w}$ is the wall shear stress, and $u$ is the streamwise $x$ velocity, $x$ is the length of the flow tube, $Q$ is the flow rate [36-38].

For a sinusoidal oscillation with angular frequency $\omega . Q(t)=Q_{\max } \sin (\omega t)$, Therefore, pressure loss can be expressed as

$$
\Delta P=\frac{\left(\bar{p}_{0}-\bar{p}_{x}\right)}{q_{j}}=\underbrace{\pi\left(S t \frac{x}{d}\right) \cos (\omega t)}_{\Delta p_{u}}+\underbrace{\int_{0}^{\frac{x}{d}} C_{f} d\left(\frac{x}{d}\right)}_{\Delta p_{s}}+\underbrace{2 \int_{-\frac{1}{2}}^{\frac{1}{2}}\left(\frac{u_{x}^{2}-u_{0}^{2}}{\bar{u}_{j}^{2}}\right) d\left(\frac{y}{d}\right)}_{\Delta p_{m}}
$$


where $\bar{U}_{j}=\frac{2}{T} d \int_{0}^{\frac{T}{2}} Q(t) d t$ is the time- and spatial-averaged expulsion velocity. Furthermore, $S t=\frac{\omega d}{\bar{U}_{j}}$ is the Strouhal number, $d$ is the inlet or outlet diameter, $C_{f}=\frac{\tau_{\omega}}{q_{j}}$ is the skin friction coefficient, $q_{j}=0.5 \rho \bar{U}_{j}^{2}$, $\Delta p_{u}$ is the unsteady, linear inertia term, $\Delta p_{s}$ is the viscous loss, $\Delta p_{m}$ is the nonlinear minor loss.

According to the pressure loss equation

$$
\Delta P=\Delta p_{u}+\Delta p_{s}+\Delta p_{m}=\xi \frac{\rho u^{2}}{2}
$$

where $\rho$ is the fluid density, $\xi$ is the flow resistance coefficient, and $u$ is the average flow velocity.

Therefore, the flow resistance coefficient in the forward flow was derived by Equations (8) and (9), as follow

$$
\xi_{+}=\frac{\Delta P}{\frac{\rho u^{2}}{2}}=\frac{\Delta p_{u}+\Delta p_{s}+\Delta p_{m}}{\frac{\rho u^{2}}{2}}=\frac{2\left(\Delta p_{u}+\Delta p_{s}+\Delta p_{m}\right)}{\rho u^{2}}
$$

where $\Delta P$ is the forward flow pressure loss, $u$ is the forward flow average velocity.

Similarly, the flow resistance coefficient in the backwards direction is

$$
\xi_{-}=\frac{\Delta P}{\frac{\rho u^{2}}{2}}=\frac{\Delta p_{u}+\Delta p_{s}+\Delta p_{m}}{\frac{\rho u^{2}}{2}}=\frac{2\left(\Delta p_{u}+\Delta p_{s}+\Delta p_{m}\right)}{\rho u^{2}}
$$

where $\Delta P$ is the backward flow pressure loss, $u$ is the average velocity of backwards flow.

When the vibrator is working, its cross section likes a paraboloid as shown in Figure 3.

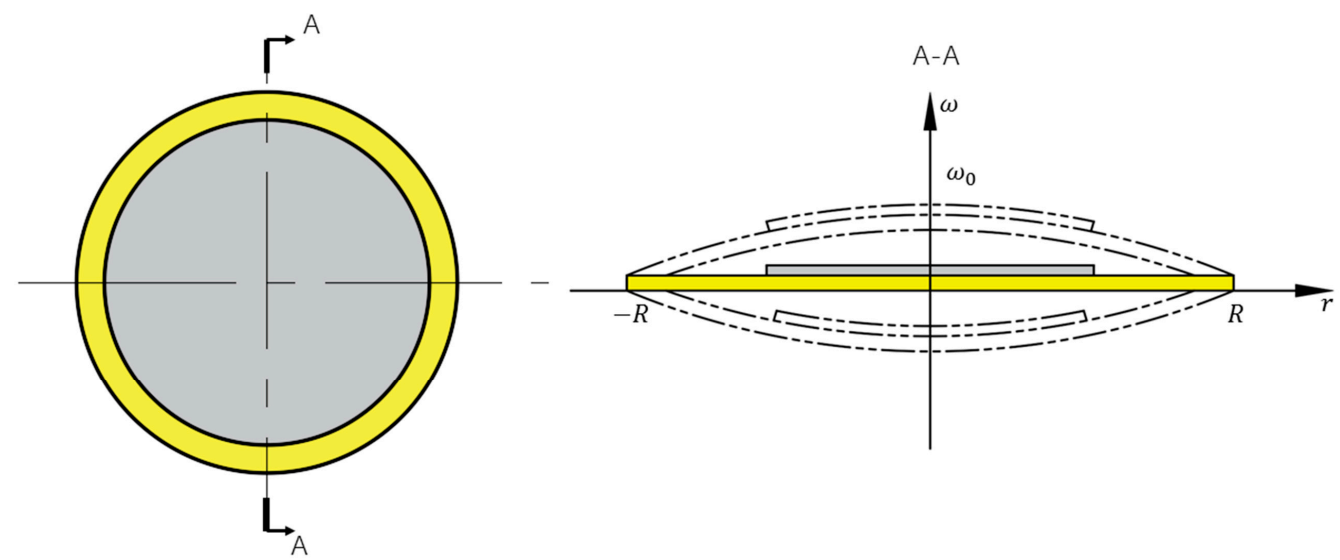

Figure 3. Piezoelectric vibrator vibration diagram.

The equation of the working section is

$$
\left\{\begin{array}{c}
\omega(r)=\omega_{0}\left(1-\frac{r^{2}}{R^{2}}\right)(|r| \leq R) \\
\omega_{0}=\frac{K d_{31} V_{(a c)}}{h^{2}} R^{2}
\end{array}\right.
$$

where $R$ is the working radius of the piezoelectric vibrator, $\omega_{0}$ is the maximum amplitude of the vibrator, $K$ is the constant which is related to the size and properties of the piezoelectric vibrator, $d_{31}$ is the piezoelectric constant, $V_{(a c)}$ is the driving voltage, $h$ is the thickness of piezoelectric vibrator [39,40].

Therefore, the volume change [41,42] caused by the PZT vibrator is

$$
\Delta V=2 \pi \int_{0}^{R} \omega_{0}\left(1-\frac{r^{2}}{R^{2}}\right) r d r=\frac{\pi K d_{31} V_{(a c)} R^{4}}{h^{2}}
$$

According to the relationship between volume change, flow rate and frequency $[43,44]$, the flow rate equation of the SLFT PZT pump is 


$$
Q_{V}=\Delta V f \frac{\xi_{-}-\xi_{+}}{2+\xi_{-}+\xi_{+}}
$$

where $Q_{V}$ is the pumping flow rate, and $f$ is the driving frequency.

\section{Flow Field Simulation}

The 3D model of the fluid area was established by Solidworks (Solidworks2016, Dassault Systemes S.A, Waltham, MA, USA), and imported into Design Modeler (ANSYS, Canonsburg, PA, USA) to mesh. The meshing model was swept with a size of $0.1 \mathrm{~mm}$, and the grids obtained were about 584,664 nodes and 569,800 elements. Finally, FLUENT (Fluent 18.1, ANSYS, Canonsburg, PA, USA) was used to conduct fluid simulation. The inlet and outlet pressure were set as $1500 \mathrm{~Pa}$ and $0 \mathrm{~Pa}$ respectively, during simulating fluid flow in forward direction. Vice versa in backward direction. The steady-state with $k-\varepsilon$ calculation model was used; and the fluid medium was room-temperature liquid water.

The pressure distribution and velocity streamline diagram of the streamlined flow tube are shown in Figures 4 and 5, respectively. It can be seen that no matter whether the fluid flowed in the forward or backward direction, the pressure distribution inside the flow tube began with increase, and then decrease. The trend of the simulated pressure results was basically consistent with that of the theoretical analysis. The trend of velocity change in the flow field was opposite to the trend of pressure change. In Figure 5, the flow rate decreased first and then increased, no matter whether the simulation was in forward or backward flow. The relationship between pressure and velocity satisfies the Bernoulli equation, and was consistent with the theoretical analysis. The simulation results of the backward flow show that a small number of vortices appeared near the maximum flow zone (Figure 5b), but there was no obvious vortex phenomenon in the forward flow (Figure 5a). It shows that the problem of vortex can be alleviated by changing the flow tendency of the streamlined flow tube.

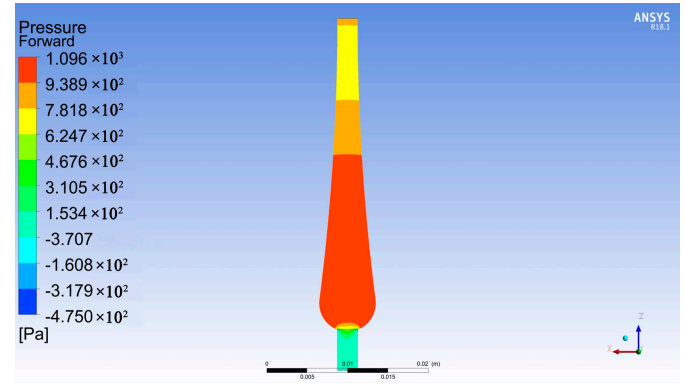

(a)

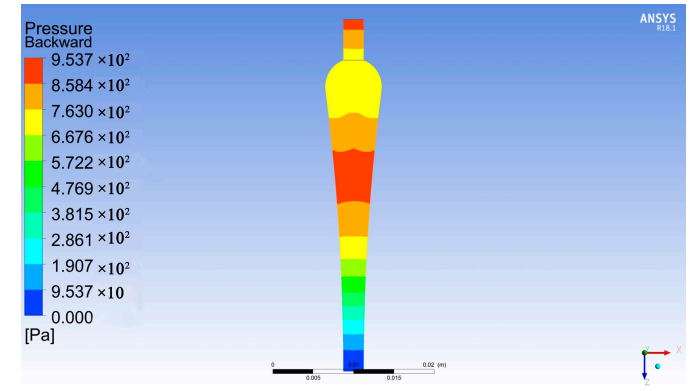

(b)

Figure 4. Pressure distribution of the streamlined flow tube. (a) Forward pressure distribution, (b) Backward pressure distribution.

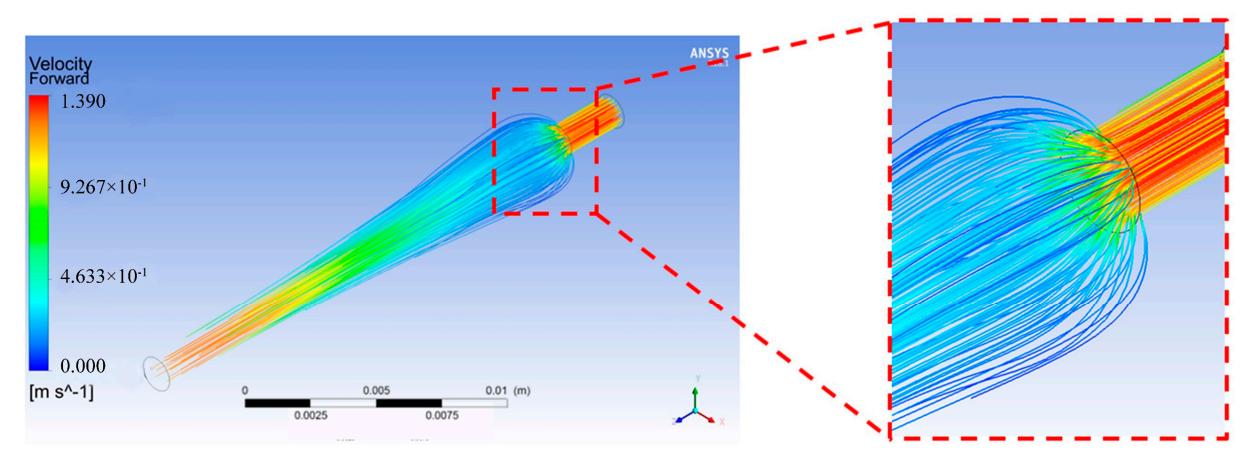

(a)

Figure 5. Cont. 


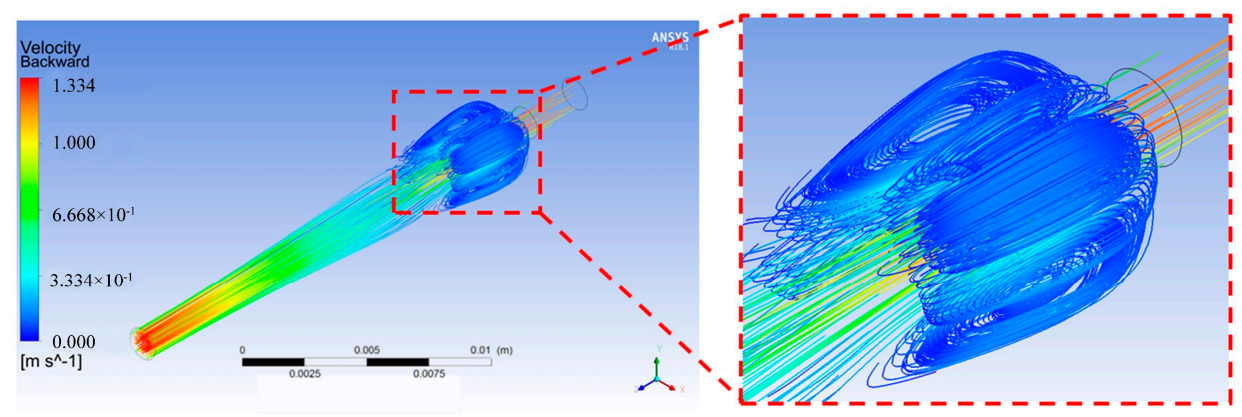

(b)

Figure 5. Velocity streamline diagram. velocity streamline.

(a) Forward velocity streamline, (b) Backward

\section{Experimental Study}

\subsection{Flow Resistance Experiment}

The flow resistance of the streamlined flow tube was measured under different pressure differences in both the forward flow direction and backward flow direction. The schematic diagram is shown in Figure 6. The left orifice of the flow tube was connected to the water storage by a silicone tube, and the water clip cut off water without obvious silicone tube deformation. The beaker was placed on the balance to collect the water outflowing from the other orifice. The pressure difference was changed by changing the height $(\mathrm{H})$ between the outlet of the streamlined tube and the liquid level of the water storage, and the total outflow mass was recorded within one minute when the water clip was removed.

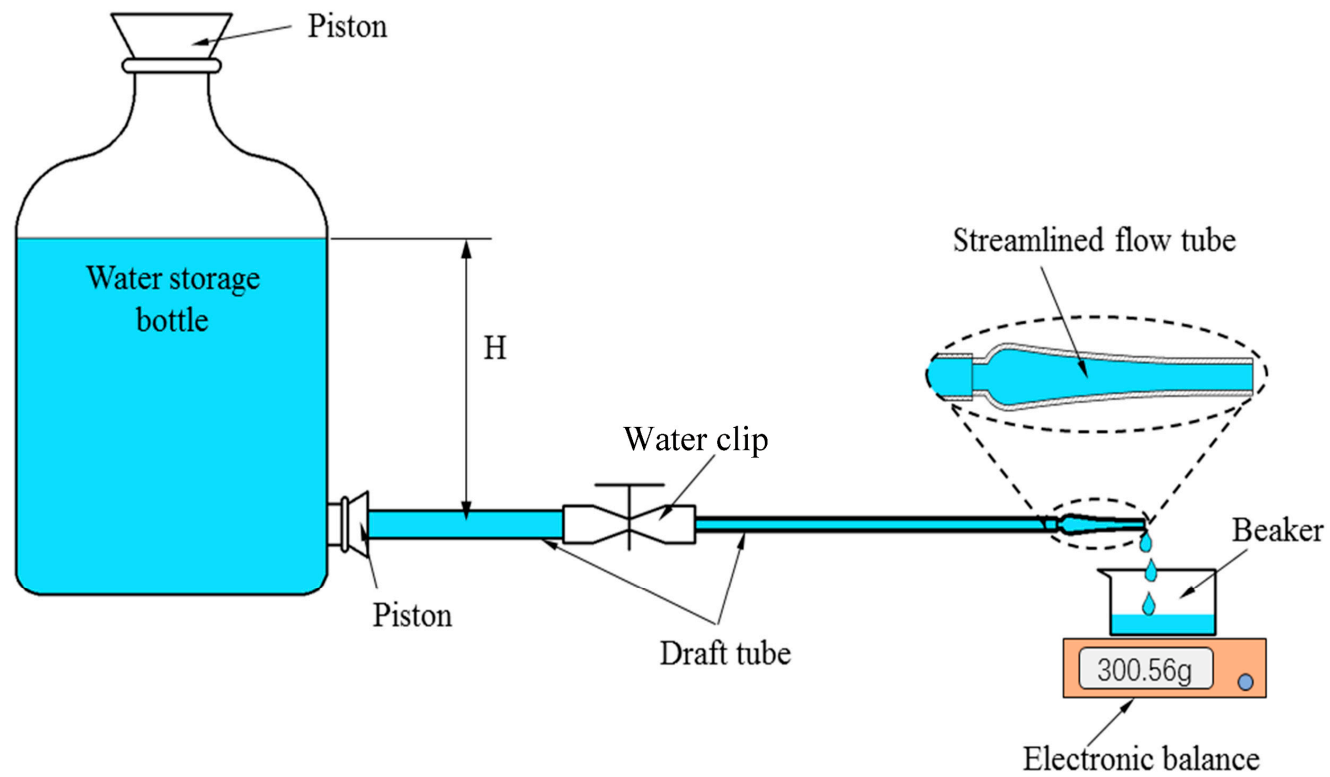

Figure 6. Flow resistance test schematic.

\subsection{Amplitude and Flow Rate Measurement}

The fluid medium used in the experiment was room-temperature liquid water. The main experimental equipment included a function signal generator (AFG1062, Tektronix, Beaverton, WA, USA), a power amplifier (HVP-300D, NJFN, Nanjing, China), an oscilloscope (DSO-X2004A, Keysight, Santa Rose, CA, USA), and a laser displacement sensor (LK-H020, Keyence, Osaka, Japan).

The amplitude of the vibrator was measured by the laser displacement sensor by focusing the laser on its center, as shown in Figure 7. The amplitude results, which would be displayed on a monitor 
screen after procession by the host, would vary with different applied voltage and frequency, which were adjusted using the signal generator and power amplifier. At the same time, during the amplitude test of the piezoelectric vibrator, liquid was injected into the valveless PZT pump through a syringe, to eliminate the influence of internal bubbles on the experiment.

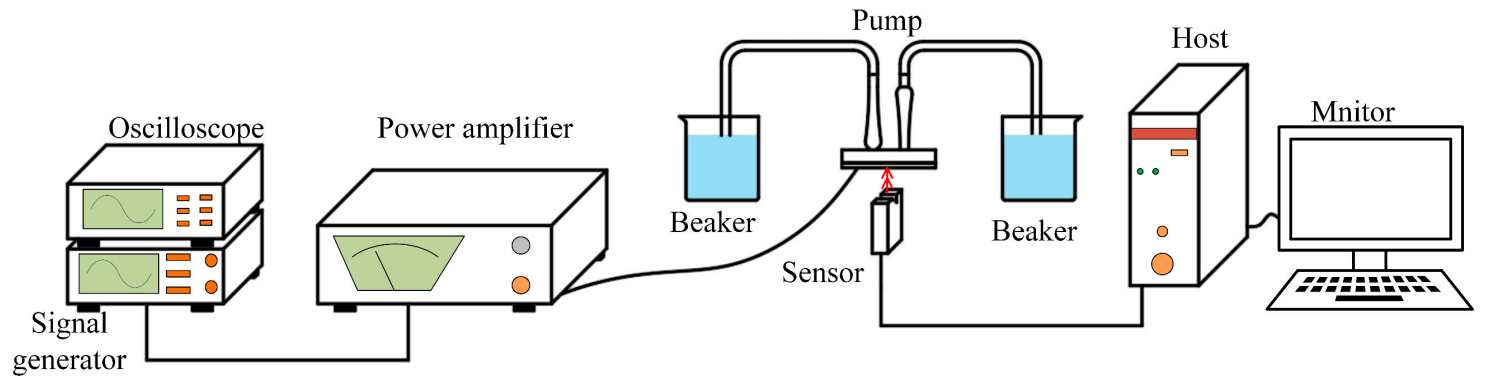

Figure 7. Schematic diagram of piezoelectric oscillator amplitude detection.

The flow rate was measured using the weight increase of the beaker on the electronic balance within one minute, during pumping fluid from the left beaker to the right using the PZT pump, as shown in Figure 8. Before the flow rate measurement, we ensured that the liquid levels of the two beakers were the same using the siphon effect, and that there were no bubbles in the tube. The actual flow rate measuring system is shown in Figure 9. To ensure the accuracy of the measurement, we quickly injected the liquid into the valveless PZT pump to elimainate the internal bubbles.

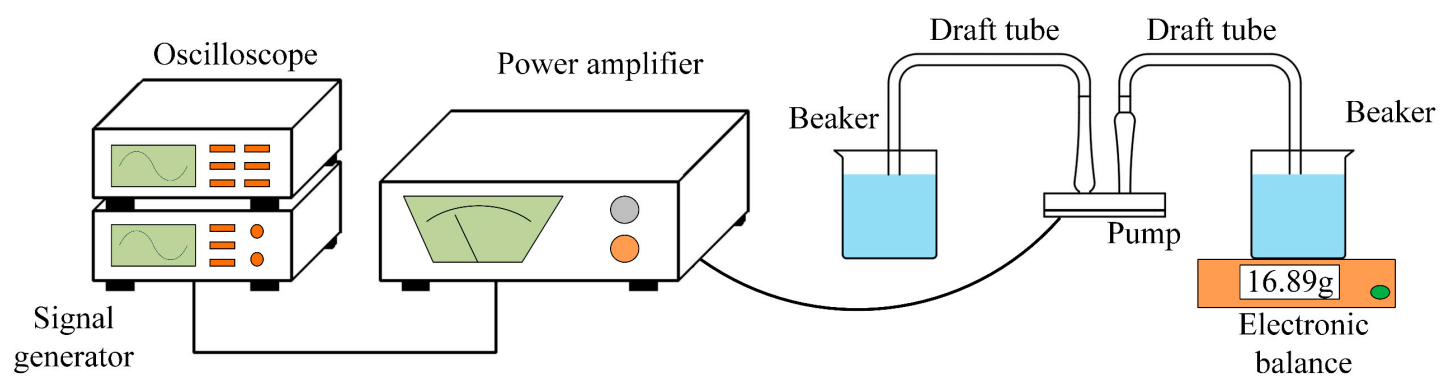

Figure 8. Schematic diagram of the flow device.

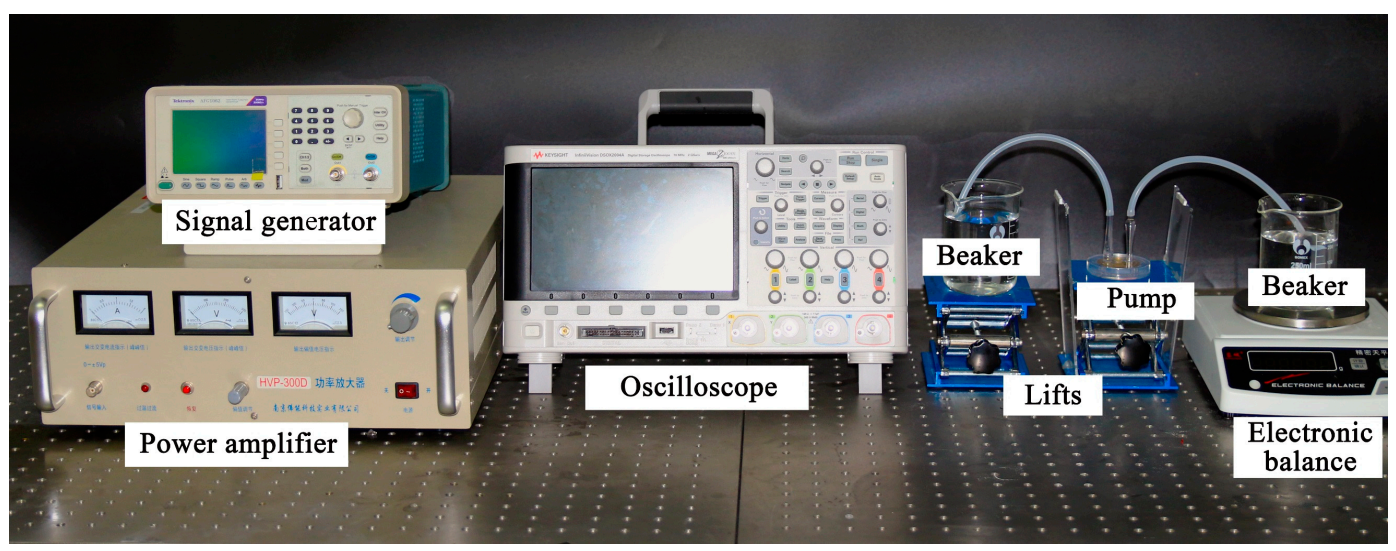

Figure 9. Traffic test platform.

\section{Results and Discussion}

The experimental and simulating flow rate results of the flow tube, under four different orifice pressure differentials of $1000 \mathrm{~Pa}, 1200 \mathrm{~Pa}, 1400 \mathrm{~Pa}$, and $1600 \mathrm{~Pa}$, were determined for fluid flow in forward and backward directions. According to the flow resistance test schematic diagram, shown 
in Figure 6, the pressure differentials can be adjusted by changing the liquid level height $\mathrm{H}$ between liquid level and flow pipes. The relationship between liquid level height and pressure difference is

$$
P=\rho g H
$$

where $P$ is the pressure difference between liquid level and flow tube, $\rho$ is the liquid density, $g$ is the gravitational acceleration.

The corresponding detail values are shown in Table 3.

Table 3. The relationship between pressure difference and liquid level.

\begin{tabular}{cccc}
\hline Density $(\rho)$ & $\begin{array}{c}\text { Gravitational } \\
\text { Acceleration }(g)\end{array}$ & Pressure Difference $(P)$ & Liquid Level Height $(\boldsymbol{H})$ \\
\hline & & $1000 \mathrm{~Pa}$ & $0.102 \mathrm{~m}$ \\
& & $1200 \mathrm{~Pa}$ & $0.122 \mathrm{~m}$ \\
& $9.8 \mathrm{~N} / \mathrm{kg}$ & $1400 \mathrm{~Pa}$ & $0.143 \mathrm{~m}$ \\
& & $1600 \mathrm{~Pa}$ & $0.163 \mathrm{~m}$ \\
\hline
\end{tabular}

The experimental and simulated flow rate results, under different pressure differences, are shown in Figure 10. As the pressure difference increased, the flow rate increased. Under any pressure difference, the forward flow rate was always greater than the backward flow rate, which proves that the forward flow resistance of the flow tube is less than the backward. According to Figure 10, the trend of the forward and backward flow rate of the streamlined flow tube obtained by simulation was basically consistent with that by experiment. However, the simulated flow rate was larger than the experimental flow rate, as there was significant pressure loss in the peripheral pipe connection system in the actual experiment.

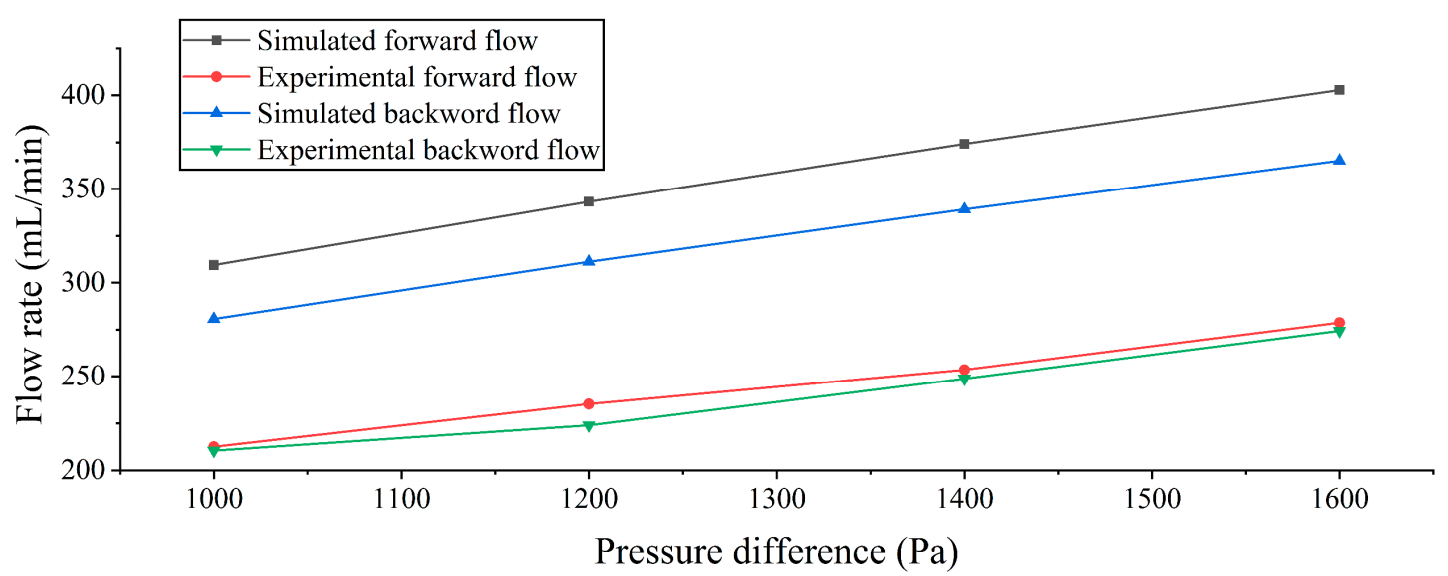

Figure 10. Flow resistance experiment and simulation.

The amplitude-frequency relationship of the SLFT PZT pump, form 40-120 V with a step of $20 \mathrm{~V}$, is shown in Figure 11. It can be seen that the amplitude of the center point of the vibrator was positively correlated with the voltage, and these maximum amplitudes were $0.062 \mathrm{~mm}(40 \mathrm{~V}, 10 \mathrm{~Hz}), 0.095 \mathrm{~mm}$ $(60 \mathrm{~V}, 10 \mathrm{~Hz}), 0.136 \mathrm{~mm}(80 \mathrm{~V}, 10 \mathrm{~Hz}), 0.217 \mathrm{~mm}(100 \mathrm{~V}, 11 \mathrm{~Hz})$, and $0.228 \mathrm{~mm}(120 \mathrm{~V}, 10 \mathrm{~Hz})$, as shown in Figure 11. In the low frequency region (1-25Hz), firstly, the amplitude of the piezoelectric vibrator increased with the increasing frequency $(1-10 \mathrm{~Hz})$, then, when the amplitude increased to a certain value, the amplitude decreased with the increasing frequency $(11-25 \mathrm{~Hz})$, and was approximately zero after $25 \mathrm{~Hz}$. In the high frequency region $(>25 \mathrm{~Hz})$, the amplitude decayed with increasing frequency and was accompanied by a few amplitude peak points. These peak points were related to the characteristic properties of the piezoelectric vibrator [16]. 


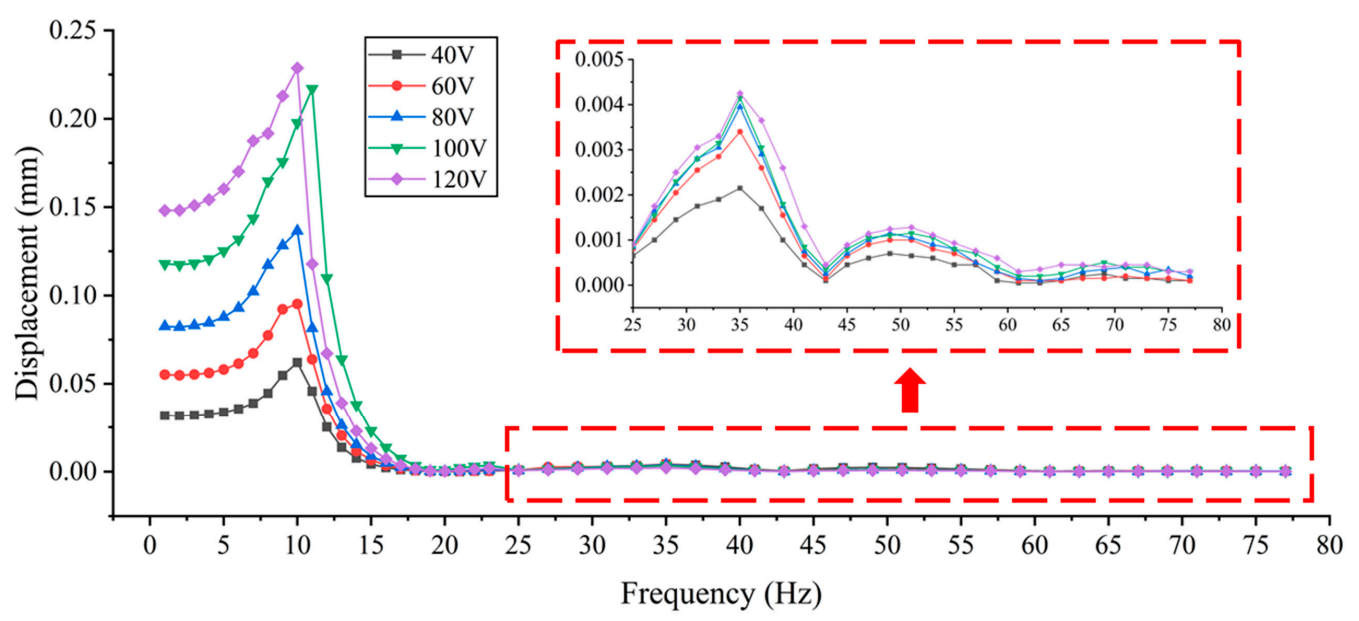

Figure 11. The amplitude results of the vibrator.

The measured flow-rate-frequency relationships of the SLFT PZT pump at different voltages are shown in Figure 12. It is obvious that the flow rate was positively related to the voltage, and that the overall trends of flow rate were basically consistent with the amplitude-frequency trend. The output flow rate increased with the driving frequency increase, before $11 \mathrm{~Hz}$, and the output flow decreased with the frequency increase after $11 \mathrm{~Hz}$. After the frequency reached $35 \mathrm{~Hz}$, the output flow remained zero. These maximum flow rates of different driving voltages were $4.79 \mathrm{~mL} / \mathrm{min}$ $(40 \mathrm{~V}, 10 \mathrm{~Hz}), 7.61 \mathrm{~mL} / \mathrm{min}(60 \mathrm{~V}, 10 \mathrm{~Hz}), 10.5 \mathrm{~mL} / \mathrm{min}(80 \mathrm{~V}, 10 \mathrm{~Hz}), 16.45 \mathrm{~mL} / \mathrm{min}(100 \mathrm{~V}, 11 \mathrm{~Hz})$ and $17.01 \mathrm{~mL} / \mathrm{min}(120 \mathrm{~V}, 10 \mathrm{~Hz})$ respectively. The low frequency region $(1-23 \mathrm{~Hz})$, where the maximum flow rate of different voltages occurred, was exactly the same as the frequency region where the maximum amplitude appeared, indicating that the pumping flow rate of the SLFT PZT pump is closely related to the vibration characteristics of the piezoelectric vibrator. In the high frequency region $(>25 \mathrm{~Hz})$, the pumping flow rate did not show more peak points as the amplitude changed. This is because the amplitude was so small; the pressure difference caused by the volume change cannot noticeably manipulate the liquid in the pump chamber.

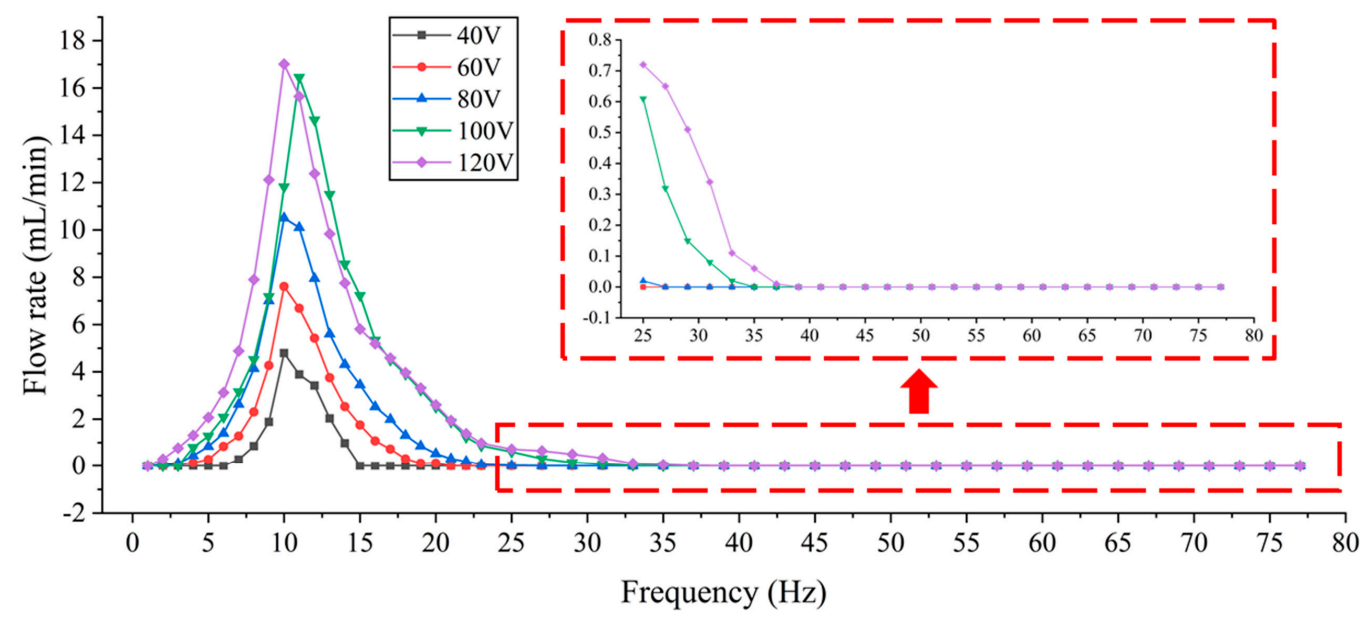

Figure 12. The flow rate results of the pump.

The local enlarged displacement-frequency relationship and flow-rate-frequency relationship are shown in the dash line rectangle $(25-80 \mathrm{~Hz})$ in Figures 11 and 12 respectively. It can be clearly seen that when the working frequency is higher than $25 \mathrm{~Hz}$, the vibration amplitude and the flow rate are rather small (less than $4 \mu \mathrm{m}$ and $0.8 \mathrm{~mL} / \mathrm{min}$, respectively) and approach zero fast. When 
the working frequency is about $10 \mathrm{~Hz}$, the vibration amplitude and flow rate reach the maximum at different voltages. Thus, we assume that this phenomenon is caused by pump system resonance.

According to the literature $[45,46]$ and the characteristics of the piezoelectric vibrator, another resonance may exist in the high-frequency region (around $1.8 \mathrm{kHz}-2.1 \mathrm{kHz}$ ). By studying the amplitude of the piezoelectric vibrator, it was found that the amplitude decreases with the frequency increase, as shown in Figure 11, and the amplitude transformation period also decreases. Although there may be another resonance in the high frequency region, the resonance amplitude in the high frequency region will be smaller than the first peak. The working medium of the piezoelectric pump is liquid, and when compared with gas, liquid has the characteristics of high density, high viscosity and incompressibility. The amplitude of the piezoelectric vibrator in the high frequency region is so small that it would cause a small change in the pump chamber volume, and that the pressure difference generated is not enough to make up for the pressure loss caused by the liquid discharged from the pump. Therefore, there is little flow rate output in the high frequency region.

According to the theoretical analysis, the resistance coefficients of forward and backward flow were obtained by simulation and substituted into Equations (10)-(12). The amplitudes were measured by laser displacement sensor and input into Equation (13), thereby calculating the theoretical flow rate. The theoretical flow rate was calculated by the amplitude of $100 \mathrm{~V}$ and $120 \mathrm{~V}$, whose results were compared with the experiment, as shown in Figure 13.

It can be seen that the maximum theoretical flow was $16.89 \mathrm{~mL} / \mathrm{min}(100 \mathrm{~V}, 10 \mathrm{~Hz})$ and $18.75 \mathrm{~mL} / \mathrm{min}$ $(120 \mathrm{~V}, 9 \mathrm{~Hz})$, respectively, and the maximum experimental flow rate was $16.45 \mathrm{~mL} / \mathrm{min}(100 \mathrm{~V}, 11 \mathrm{~Hz})$ and $17.01 \mathrm{~mL} / \mathrm{min}(120 \mathrm{~V}, 10 \mathrm{~Hz})$, respectively. In the low frequency region $(1-25 \mathrm{~Hz})$, the theoretical flow rate was consistent with the experimental flow. In the high frequency region $(>25 \mathrm{~Hz})$, the experimental flow was close to zero, and the theoretical flow decreased in fluctuation corresponding to the fluctuation of amplitude, further proving the direct relationship between amplitude and pumping flow rate. By comparison, it was found that the frequency of maximum flow rate in the experiment lagged behind the frequency of maximum flow rate by theory. Several reasons account for the frequency delay, when compared with the theoretical results, and are illustrated as follows:

1. The vibrator was attached to pump chamber by silica gel bonding, which indirectly increases the volume and reduces the volume change quantity of the pump chamber to some extent.

2. The connection between the flow tube and the pump body is prone to pressure loss, resulting in increased internal pressure loss and decreased pumping efficiency.

3. The viscosity of the experimental water is inevitable, which also causes fluid flowing hysteresis.

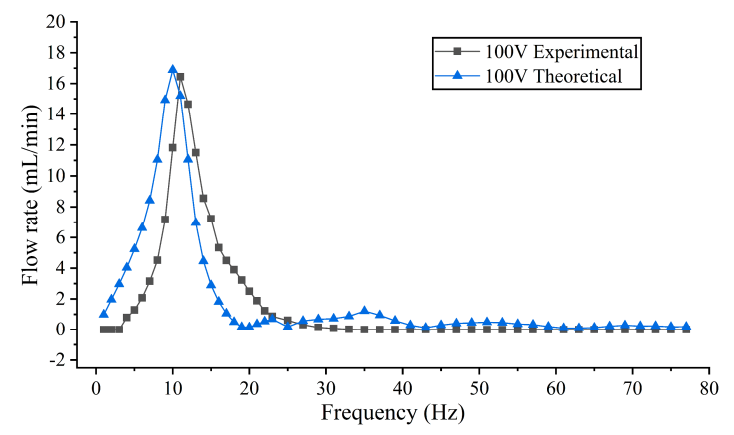

(a)

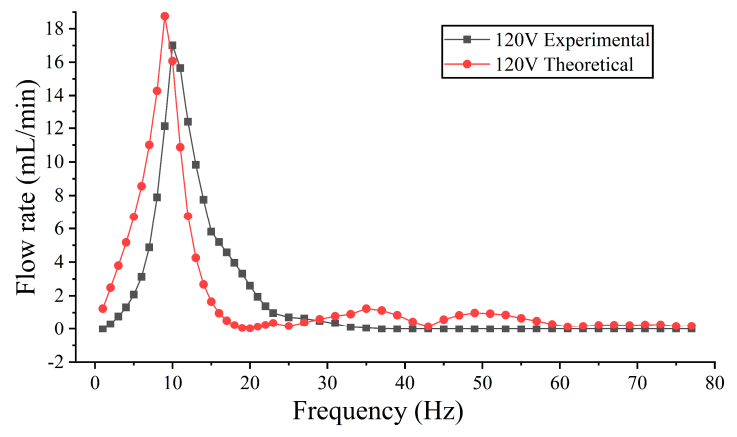

(b)

Figure 13. The theoretical and experimental flow rate at 100 and $120 \mathrm{~V}$. (a) The flow rate at $100 \mathrm{~V}$, (b) The flow rate at $120 \mathrm{~V}$.

\section{Conclusions}

Based on the existing research, a streamlined flow tube valveless piezoelectric pump (SLFT PZT pump) was fabricated and analyzed. Firstly, the structural and working principle of the flow tube were analyzed. At the same time, the theoretical flow rate and flow resistance coefficient equations were 
established. Secondly, the pressure and velocity field of the flow tube were further simulated, and the results fit the theoretical well, proving that the flow tube has the ability to modify the single flow trend and improve the flow stability. Thirdly, the amplitude-frequency relationships of the piezoelectric vibrator, and the flow-rate-frequency relationships of the SLFT PZT pump were tested at 40,60, 80, 100 and $120 \mathrm{~V}$. The results show that both relations trends are basically the same, indicating that the flow rate was highly related to the vibrator amplitude. Finally, in order to verify the correctness of the theoretical analysis, the flow resistance coefficient of the flow tube, under $100 \mathrm{~V}$ and $120 \mathrm{~V}$, was substituted into the flow rate equation to obtain the theoretical flow rate, and the results were basically the same as the experimental results.

Author Contributions: J.Z. conceived and designed the experiments; M.T. performed the experiments; J.Z. analyzed the data; M.T. and Q.B. contributed reagents/materials/analysis tools; Ming Tang wrote the paper; J.H. Q.N. and C.C. proofread the paper, C.W. revised the spelling and grammar of the text.

Funding: The research work presented in this paper is supported by National Natural Science Foundation of China: 51375227, Scientific Research Project in Municipal Colleges and of Universities of Guangzhou: 1201610315.

Conflicts of Interest: The authors declare that no conflicts of interest exist.

\section{References}

1. Ji, X.P.; Hou, Y.Q.; Chen, Y.; Zhen, Y.K. Attenuation of acoustic wave excited by piezoelectric aggregate in asphalt pavement and its application to monitor concealed cracks. Constr. Build. Mater. 2019, 216, 58-67. [CrossRef]

2. Mathew, A.T.; Liu, C.; Ng, T.Y.N.; Koh, S.J.A. A high energy dielectric-elastomer-amplified piezoelectric (DEAmP) to harvest low frequency motions. Sens. Actuator A Phys. 2019, 294, 61-72. [CrossRef]

3. Nikoei, S.; Hassani, B. Isogeometric analysis of laminated smart shell structures covered with piezoelectric sensors and actuators using degenerated shell formulation. J. Intell. Mater. Syst. Struct. 2019, 30, 1913-1931. [CrossRef]

4. Calahorra, Y.; Husmann, A.; Bourdelain, A.; Kim, W.; Vukajlovic-Plestina, J.; Boughey, C.; Jing, Q.S.; Morral, A.F.I.; Kar-Narayan, S. Highly sensitive piezotronic pressure sensors based on undoped GaAs nanowire ensembles. J. Phys. D Appl. Phys. 2019, 52, 7. [CrossRef]

5. Nabavi, S.; Zhang, L. Nonlinear Multi-Mode Wideband Piezoelectric MEMS Vibration Energy Harvester. IEEE Sens. J. 2019, 19, 4837-4848. [CrossRef]

6. Deng, W.; Yang, T.; Jin, L.; Yan, C.; Huang, H.; Chu, X.; Wang, Z.; Xiong, D.; Tian, G.; Gao, Y.; et al. Cowpea-structured PVDF/ZnO nanofibers based flexible self-powered piezoelectric bending motion sensor towards remote control of gestures. Nano Energy 2019, 55, 516-525. [CrossRef]

7. Lim, S.; Son, D.; Kim, J.; Lee, Y.B.; Song, J.K.; Choi, S.; Lee, D.J.; Kim, J.H.; Lee, M.; Hyeon, T.; et al. Transparent and Stretchable Interactive Human Machine Interface Based on Patterned Graphene Heterostructures. Adv. Funct. Mater. 2015, 25, 375-383. [CrossRef]

8. Deng, J.; Liu, Y.X.; Liu, J.K.; Xu, D.M.; Wang, Y. Development of a Planar Piezoelectric Actuator Using Bending-Bending Hybrid Transducers. IEEE Trans. Ind. Electron. 2019, 66, 6141-6149. [CrossRef]

9. Yang, L.; Wang, F.; Zhang, J.J.; Ren, W.H. Remaining useful life prediction of ultrasonic motor based on Elman neural network with improved particle swarm optimization. Measurement 2019, 143, 27-38. [CrossRef]

10. Setareh, M.; Saffar-Avval, M.; Abdullah, A. Experimental and numerical study on heat transfer enhancement using ultrasonic vibration in a double-pipe heat exchanger. Appl. Therm. Eng. 2019, 159, 13. [CrossRef]

11. Ye, Z.; Zhang, R.; Gao, M.; Deng, Z.; Gui, L. Development of a High Flow Rate 3-D Electroosmotic Flow Pump. Micromachines 2019, 10, 112. [CrossRef] [PubMed]

12. Lin, Y.-C.; Huang, Y.-H.; Chu, K.-W. Experimental and Numerical Investigation of Resonance Characteristics of Novel Pumping Element Driven by Two Piezoelectric Bimorphs. Appl. Sci. 2019, 9, 1234. [CrossRef]

13. Park, S.S.; Lee, J.S.; Lee, D.E.; Lee, J.C. Measurement of Unit Weight of Dry Sand Using Piezoelectric Sensor. Appl. Sci. 2018, 8, 8. [CrossRef]

14. Qi, B.; Kong, Q.; Qian, H.; Patil, D.; Lim, I.; Li, M.; Liu, D.; Song, G. Study of Impact Damage in PVA-ECC Beam under Low-Velocity Impact Loading Using Piezoceramic Transducers and PVDF Thin-Film Transducers. Sensors 2018, 18, 671. [CrossRef] [PubMed] 
15. Fuchs, O.; Fouillet, Y.; Maubert, S.; Cochet, M.; Chabrol, C.; David, N.; Médal, X.; Campagnolo, R. A novel volumetric silicon micropump with integrated sensors. Microelectron. Eng. 2012, 97, 375-378. [CrossRef]

16. Bao, Q.; Zhang, J.; Tang, M.; Huang, Z.; Lai, L.; Huang, J.; Wu, C. A Novel PZT Pump with Built-in Compliant Structures. Sensors 2019, 19, 1301. [CrossRef]

17. Cazorla, P.-H.; Fuchs, O.; Cochet, M.; Maubert, S.; Le Rhun, G.; Robert, P.; Fouillet, Y.; Defay, E. Piezoelectric micro-pump with PZT thin film for low consumption microfluidic devices. Procedia Eng. 2014, 87, 488-491. [CrossRef]

18. Hu, B.; Yu, H.J.S. Optimal Design and Simulation of a Microsuction Cup Integrated with a Valveless Piezoelectric Pump for Robotics. Shock Vib. 2018, 2018, 1-16. [CrossRef]

19. Qu, Y.; Zhou, J.; Wu, W. Theoretical and Experimental Research on Bubble Actuated Micro-Pumps. Micromachines 2018, 9, 225. [CrossRef]

20. Stemme, E.; Stemme, G.J.S.; Physical, A.A. A valveless diffuser/nozzle-based fluid pump. Sens. Actuators A Phys. 1993, 39, 159-167. [CrossRef]

21. Zhang, J.-H.; Wang, Y.; Huang, J. Advances in Valveless Piezoelectric Pump with Cone-shaped Tubes. Chin. J. Mech. Eng. 2017, 30, 766-781. [CrossRef]

22. Iverson, B.D.; Garimella, S.V. Recent advances in microscale pumping technologies: A review and evaluation. Microfluid. Nanofluid. 2008, 5, 145-174. [CrossRef]

23. He, X.H.; Cai, S.C.; Deng, Z.D.; Yang, S. Experimental and numerical study of flow characteristics of flat-walled diffuser/nozzles for valveless piezoelectric micropumps. Proc. Inst. Mech. Eng. Part C J. Eng. Mech. Eng. Sci. 2017, 231, 2313-2326. [CrossRef]

24. He, X.; Zhu, J.; Zhang, X.; Xu, L.; Yang, S. The analysis of internal transient flow and the performance of valveless piezoelectric micropumps with planar diffuser/nozzles elements. Microsyst. Technol. 2015, 23, 23-37. [CrossRef]

25. Forster, F.K.; Bardell, R.L.; Afromowitz, M.A.; Sharma, N.R.; Blanchard, A. Design, fabrication and testing of fixed-valve micro-pumps. ASME-PUBLICATIONS-FED 1995, 234, 39-44.

26. Gamboa, A.R.; Morris, C.J.; Forster, F.K. Improvements in Fixed-Valve Micropump Performance through Shape Optimization of Valves. J. Fluids Eng. 2005, 127, 339-346. [CrossRef]

27. Huang, J.; Zhang, J.; Wang, S.; Liu, W. Analysis of the Flow Rate Characteristics of Valveless Piezoelectric Pump with Fractal-like Y-shape Branching Tubes. Chin. J. Mech. Eng. 2014, 27, 628-634. [CrossRef]

28. Zhang, J.; Li, Y.; Xia, Q. Analysis of the pump volume flow rate and tube property of the piezoelectric valveless pump with Y-shape tubes. Chin. J. Mech. Eng. 2007, 43, 136-141. [CrossRef]

29. Munas, F.R.; Melroy, G.; Abeynayake, C.B.; Chathuranga, H.L.; Amarasinghe, R.; Kumarage, P.; Dau, V.T.; Dao, D.V. Development of PZT Actuated Valveless Micropump. Sensors 2018, 18, 1302. [CrossRef] [PubMed]

30. He, L.; Zhao, D.; Li, W.; Xu, Q.; Cheng, G. Performance analysis of valveless piezoelectric pump with dome composite structures. Rev. Sci. Instrum. 2019, 90, 065002. [CrossRef]

31. Huang, X.Y.; Wang, S.S.; Yang, C. Single-Nozzle Micropumps. In ASME 2009 7th International Conference on Nanochannels, Microchannels, and Minichannels; American Society of Mechanical Engineers: New York, NY, USA, 2009; pp. 717-721.

32. Wang, S.S.; Huang, X.Y.; Yang, C. Valveless micropump with acoustically featured pumping chamber. Microfluid. Nanofluid. 2010, 8, 549-555. [CrossRef]

33. Schobeiri, M.T. Boundary Layer Theory. In Fluid Mechanics for Engineers: A Graduate Textbook; Springer: Berlin/Heidelberg, Germany, 2010; 11p.

34. Samokhin, V. Mathematical Models in Boundary Layer Theory; Routledge: New York, NY, USA, 2018; pp. $120-250$.

35. Cao, H.L.; Guo, W.; Li, X.H.; Zhou, Y. Effect of Micro-bubbles on a Turbulent Boundary Layer; Springer: Singapore, 2017; pp. 137-142.

36. Raju, R.; Gallas, Q.; Mittal, R.; Cattafesta, L. Scaling of pressure drop for oscillatory flow through a slot. Phys. Fluids 2007, 19, 078107. [CrossRef]

37. Chiatto, M.; Capuano, F.; Luca, L.D. Numerical and experimental characterization of a double-orifice synthetic jet actuator. Meccanica 2018, 53, 1-14. [CrossRef]

38. Gallas, Q.; Holman, R.; Nishida, T.; Carroll, B.; Sheplak, M.; Cattafesta, L. Lumped Element Modeling of Piezoelectric-Driven Synthetic Jet Actuators. AIAA J. 2002, 41, 11.

39. Ullmann, A. The piezoelectric valve-less pump-Performance enhancement analysis. Sens. Actuators A Phys. 1998, 69, 97-105. [CrossRef] 
40. Kan, J.W.; Cao, R.Q.; Yang, Z.G.; Cheng, G.M. Structure Design and Performance Analysis of a Piezoelectric Pump. Piezoelectr. Acoustooptics 2002, 24, 368-371.

41. Cheng, G.; Suzuki, K. Research on the Cavity Volume Fluctuations of Piezoelectric Pump. Piezoelectr. Acoustooptics 1998, 20, 389-392.

42. Zhang, J.; Wang, S.; Yang, D. A study on a valveless, piezoelectric diffuser/nozzle-based fluid pump: Analysis of vibration and the changed amount of the chamber volume. Piezoelectr. Acoustooptics 1999, 21, 457-460.

43. Zhang, J.H.; Wang, S.Y. Study of Piezoelectric Valveless Diffuser/Nozzle-based Fluid Pump: One-way Flow Principle and the Pump Flow. Piezoelectr. Acoustooptics 2001, 23, $23-25$.

44. Yan, Q.; Zhang, J.; Huang, J.; Wang, Y.J.S. The Effect of Vibration Characteristics on the Atomization Rate in a Micro-Tapered Aperture Atomizer. Sensors 2018, 18, 934. [CrossRef]

45. An, D.; Ming, Y.; Zhuang, X.; Yang, T.; Fan, M.; Dong, Z. Dual traveling wave rotary ultrasonic motor with single active vibrator. Appl. Phys. Lett. 2017, 110, 143507. [CrossRef]

46. Chiatto, M.; Palumbo, A.; de Luca, L. Design approach to predict synthetic jet formation and resonance amplifications. Exp. Therm. Fluid Sci. 2019, 107, 79-87. [CrossRef]

(C) 2019 by the authors. Licensee MDPI, Basel, Switzerland. This article is an open access article distributed under the terms and conditions of the Creative Commons Attribution (CC BY) license (http://creativecommons.org/licenses/by/4.0/). 